\title{
PISCO: the Parallel Imager for Southern Cosmology Observations
}

\section{Citation}

Stalder, Brian, et al. 2014. PISCO: the Parallel Imager for Southern Cosmology Observations. In the Proceedings of Ground-based and Airborne Instrumentation for Astronomy V (SPIE 9147): July 8, 2014. doi:10.1117/12.2054933.

\section{Published Version}

doi:10.1117/12.2054933

\section{Permanent link}

http://nrs.harvard.edu/urn-3:HUL.InstRepos:20569064

\section{Terms of Use}

This article was downloaded from Harvard University's DASH repository, and is made available under the terms and conditions applicable to Other Posted Material, as set forth at http:// nrs.harvard.edu/urn-3:HUL.InstRepos:dash.current.terms-of-use\#LAA

\section{Share Your Story}

The Harvard community has made this article openly available.

Please share how this access benefits you. Submit a story.

\section{Accessibility}




\title{
PISCO: the Parallel Imager for Southern Cosmology Observations
}

\author{
Brian Stalder ${ }^{a}$, Antony A. Stark ${ }^{a}$, Stephen M. Amato ${ }^{a}$, John Geary $^{a}$, Stephen A. Shectman ${ }^{b}$ \\ Christopher W. Stubbs ${ }^{c, a}$, and Andrew Szentgyorgyi ${ }^{a}$ \\ ${ }^{a}$ Harvard-Smithsonian Center for Astrophysics, 60 Garden St, Cambridge, MA, USA; \\ ${ }^{b}$ Carnegie Observatories, 813 Santa Barbara St, Pasadena, CA, USA; \\ ${ }^{c}$ Harvard University Department of Physics, 17 Oxford St, Cambridge, MA, USA
}

\begin{abstract}
We present the design and lab performance of the Parallel Imager for Southern Cosmology Observations (PISCO), a photometer for the $6.5 \mathrm{~m}$ diameter Magellan telescopes that produces $g^{\prime}, r^{\prime}, i^{\prime}$, and $z^{\prime}$ band images simultaneously within a 9 arcminute field of view. This design provides efficient follow-up observations of faint sources, particularly galaxy clusters and supernovae. Simultaneous imaging speeds the observing cadence by at a factor of $\sim 3$ (including optical losses) compared to other photometric imagers. Also, the determination of color (flux ratio between bands) is relatively immune to time variations in gray opacity due to clouds, so observations can proceed in less than optimal conditions. First light is expected in September 2014.
\end{abstract}

Keywords: Optical photometry, Magellan telescopes, CCD, Multi-band imaging

\section{INTRODUCTION}

The Parallel Imager for Southern Cosmology Observations (PISCO) is designed to be a rapid and efficient multiband imager for follow-up of faint sources identified by current large surveys. PISCO is a photometric camera that comprises four focal planes with a common shutter, capable of obtaining simultaneous images over a 9 arcminute feld of view in 4 optical passbands $(400-550 \mathrm{~nm}, 550-700 \mathrm{~nm}, 700-850 \mathrm{~nm}$, and $820-1100 \mathrm{~nm}$, essentially the Sloan Digital Sky Survey $g^{\prime}, r^{\prime}, i^{\prime}$, and $z^{\prime}$ bands). The instrument can be mounted on any available f/11 focus port on the Magellan telescopes.

The paper is organized as follows: in Section 2 we describe the primary motivation of building PISCO. The optical design is detailed in Section 3 followed by a description of the mechanical systems in Section 4 . In Section 6 we present the overall stucture of the software that controls PISCO and quickly analyzes the data for on-telescope optimization of the observing cadence. Finally, we report some laboratory performance data in Section 7 .

\section{KEY SCIENCE PROGRAM}

PISCO is designed to rapidly and effciently take the first images and determine redshifts for SZ-selected clusters discovered by SPT. ${ }^{1}$ Its control software (descibed in Section 6) includes a near real-time data-reduction pipeline that can determine if a cluster has been detected and the data are suffcient to determine a redshift; if so, it moves automatically to the next cluster target, performing telescope overhead operations only when necessary.

Multiple authors have demonstrated that these four bands are well-suited to measuring photometric redshifts over the desired redshift range. Padmanabhan et al. used only 3 SDSS bands $\left(g^{\prime}, r^{\prime}\right.$, and $\left.i^{\prime}\right)$ and obtained errors in cluster redshifts $\mathrm{E}(\mathrm{z}) \sim 0.02$ for redshifts out to $0.8 .^{2}$ Bahcall et al. used the full SDSS filter set to achieve errors in cluster redshifts $\mathrm{E}(\mathrm{z}) \sim 0.02$ for $z<0.3$ and $\mathrm{E}(\mathrm{z}) \sim 0.03$ for $z<1.0 .^{3}$ Gladders et al. obtained $\mathrm{E}(\mathrm{z}) \sim 0.1$ for $1.0<z<1.5$ in a two-color survey with $R_{C}$ and $\mathrm{z}$ bands only. ${ }^{4}$ Our enhanced CCD QE in the $z^{\prime}$ band will similarly allow us to extend out to redshift $z \sim 1.5$, the point at which the $4000 \AA$ break shifts beyond the

Further author information: (Send correspondence to B.S.)

B.S.: E-mail: bstalder@cfa.harvard.edu, Telephone: 16174967360

Ground-based and Airborne Instrumentation for Astronomy V, edited by

Suzanne K. Ramsay, lan S. McLean, Hideki Takami, Proc. of SPIE Vol. 9147

91473Y · (C) 2014 SPIE · CCC code: 0277-786X/14/\$18 - doi: 10.1117/12.2054933

Proc. of SPIE Vol. 9147 91473Y-1 


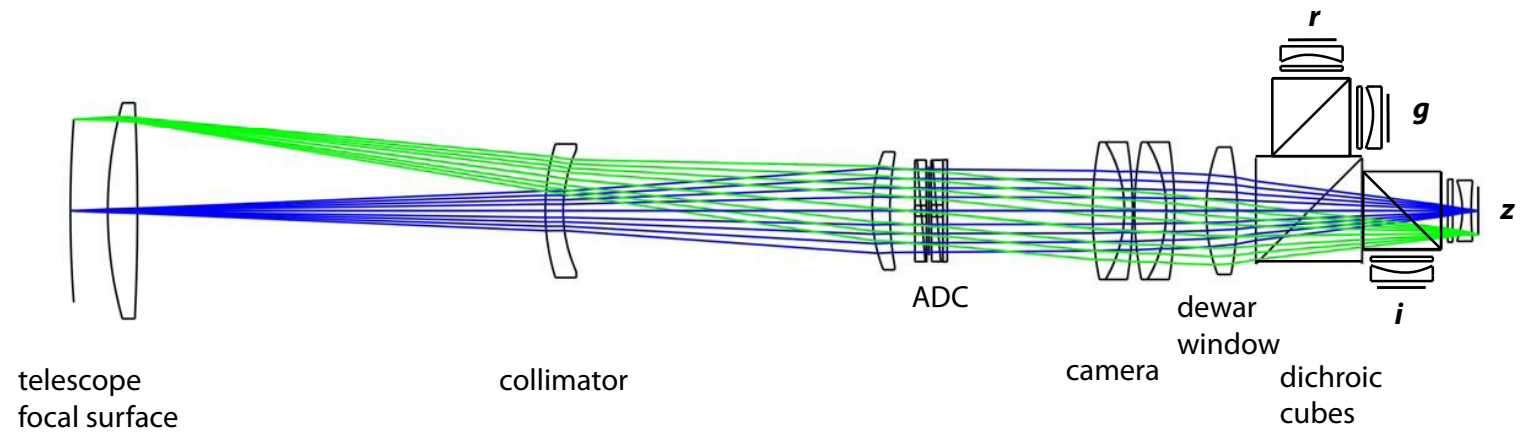

Figure 1. The optical layout of the PISCO multi-image photometer. The dichroic cubes illuminate four CCDs, each sensitive to a different passband, $g^{\prime}, r^{\prime}, i^{\prime}$, and $z^{\prime}$.

$z^{\prime}$ band. The latest performance from optical follow-up of SPT clusters ${ }^{5}$ at $z<0.9$ shows photometric redshift errors $\mathrm{E}(\mathrm{z})<0.02$, using only the $g^{\prime}, r^{\prime}, i^{\prime}$, and $z^{\prime}$ bands. PISCO's simultaneous exposures in all four bands will further reduce the systematic errors that dominate redshift determination.

Note that our goal is to determine the photometric redshift of a cluster of galaxies at a known location, as we intend to take observations pointed at the locations of candidate galaxy clusters. This is considerably more accurate and reliable than trying to measure the photometric redshift of a single isolated galaxy. We can pick the luminous red cluster members, and can use robust statistics to eliminate outliers. PISCO is very well suited to the determination of cluster redshifts using photometry. The 9 arcminute field spans $1.5 \mathrm{Mpc}$ at $z=0.3$, which is nicely matched to the size of a typical cluster. As a result of cosmological magnification, all clusters at $z>0.1$ are about this size.

\section{OPTICAL DESIGN}

The optical design consists of re-imaging optics that generate a telecentric beam which is dissected into wavelength bands by a system of dichroic surfaces embedded in prisms followed by standard dielectric filters (cf. Figure 1). The first three lens elements comprise a collimator that produces a parallel beam from each star. This is followed by an atmospheric dispersion compensator (ADC) based on a rotating 2-prism design by A. Szentgyorgi. Next is a 7.5-inch square carbon-fiber blade photometric shutter designed and built by Sci-In Tech. The shutter is a photometric design: it always allows equal exposure time for every optical ray. Two identical doublets and a singlet lens that is also the cryostat window generate the telecentric beam to feed the dichroics. For each channel, a filter can then be placed in the color-separated beam either to sharpen the bandpass edges of the standard filter set, or to provide up to four simultaneous narrow bandpasses. For example, the $z^{\prime}$ bandpass filter could be replaced by a $z_{\text {short }}$ or $Y$ filter. Additionally, the $r^{\prime}$ filter could be replaced by an H $\alpha$ filter, while the $g^{\prime}$ filter may be replaced by an O III filter. Finally, a field lens focuses the image on each CCD. The overall optical system reduces the $\mathrm{f} / 11$ beam from the Magellan telescope to $\mathrm{f} / 3$ at the CCD surface, translating to a plate scale of 10.8 milliarcseconds $/ \mu \mathrm{m}$, and has seeing-limited performance over the full field of view (Figure 2).

\subsection{Dichroic Cubes}

The design of the dichroic system is novel, and overcomes some limitations of previous multi-band imagers. ${ }^{6-8}$ The fundamental issue of multiplexing an optical beam is that in order to avoid rays near the Brewster's angle at the dichroics, one usually puts the beam splitter in a parallel (collimated) beam. This however causes a variation in the spectral transmission/reflection with field position. Our solution is to embed the dichroics in fused silica and place them in a telecentric beam, so that the collection of rays from each star impact the dichroics over the same distribution of angles and do not go through a change in index of refraction at the dichroic interfaces. 


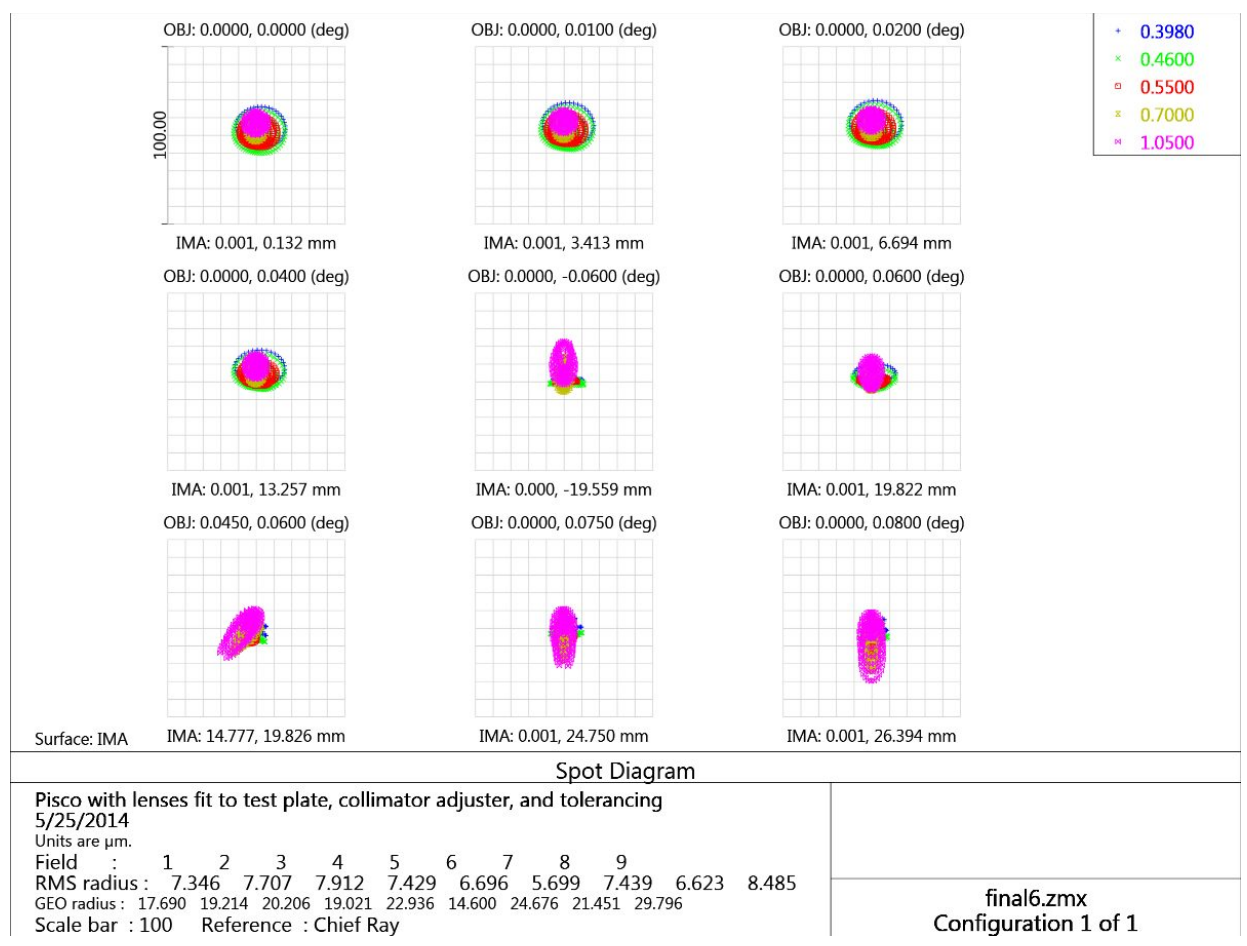

Figure 2. Spot diagram of the as-built PISCO optics. Although the optics are not diffraction-limited, spot sizes are less than the best Magellan seeing: at the CCD, 0.3 arcseconds $\simeq 28 \mu \mathrm{m}$. The CCD pixels are $10 \mu \mathrm{m}$, the size of the small squares in this diagram. Colors span the range from $398 \mathrm{~nm}$ to $1050 \mathrm{~nm}$.

\section{MECHANICAL DESIGN}

Figure 3 shows the overall mechanical layout of PISCO. The large outer box structure houses the fore-optics (collimator and $\mathrm{ADC}$ ) and the shutter as well as providing the structural stiffness for the entire instrument. The box mounts to a large flange that interfaces with the standard Magellan small guider housing and supports a cable wrap. At the far end of the main structure is the cryostat that holds the dichroics, filters, field lens, and the CCDs with associated thermal control and data readout systems. The on-board sensing and control electronics are housed in 4 boxes on each side of the main structure. The instrument is 1.88 meters long and 0.87 meters wide. The moving mechanical parts in PISCO are the ADC rotator, a 4 degree-of-freedom actuator for the collimator position, the shutter, and the cooling fans for the electronics.

\subsection{Atmospheric Dispersion Compensator (ADC)}

Atmospheric dispersion at large zenith angles can elongate the point spread function of PISCO by a few arcseconds, even within a single SDSS bandpass. To correct for this, the PISCO ADC consists of two counter-rotating cylindrically-shaped prisms made of PBL6Y and S-FSL5Y glass. The two prisms are identical, except that one has a long-pass filter (cuton at $400 \mathrm{~nm}$ ) cemented to one face. All glass surfaces and interfaces are flat, but the top and bottom planes of the prisms are not parallel - they deviate by 36 minutes of arc, so that the thickness of the higher-index PBL6Y glass is a little less than that of the S-FSL5Y. This "top angle" is critical for reducing aberrations. The bottom surface of the S-FSL5Y glass is perpendicular to the optical axis, and the prisms rotate around the optical axis. The prisms are $102 \mathrm{~mm}$ in diameter, and $20 \mathrm{~mm}$ thick. The thickness is divided equally between the two constituent glass pieces, although the thickness dimensions have a relatively large error tolerance $( \pm 0.3 \mathrm{~mm})$. The two prisms are separated by $4 \mathrm{~mm}$, and an image of the aperture is located between them. The maximum correction configuration $\left(90^{\circ}\right.$ counter-rotation) corrects for a zenith angle of $71^{\circ}$. 

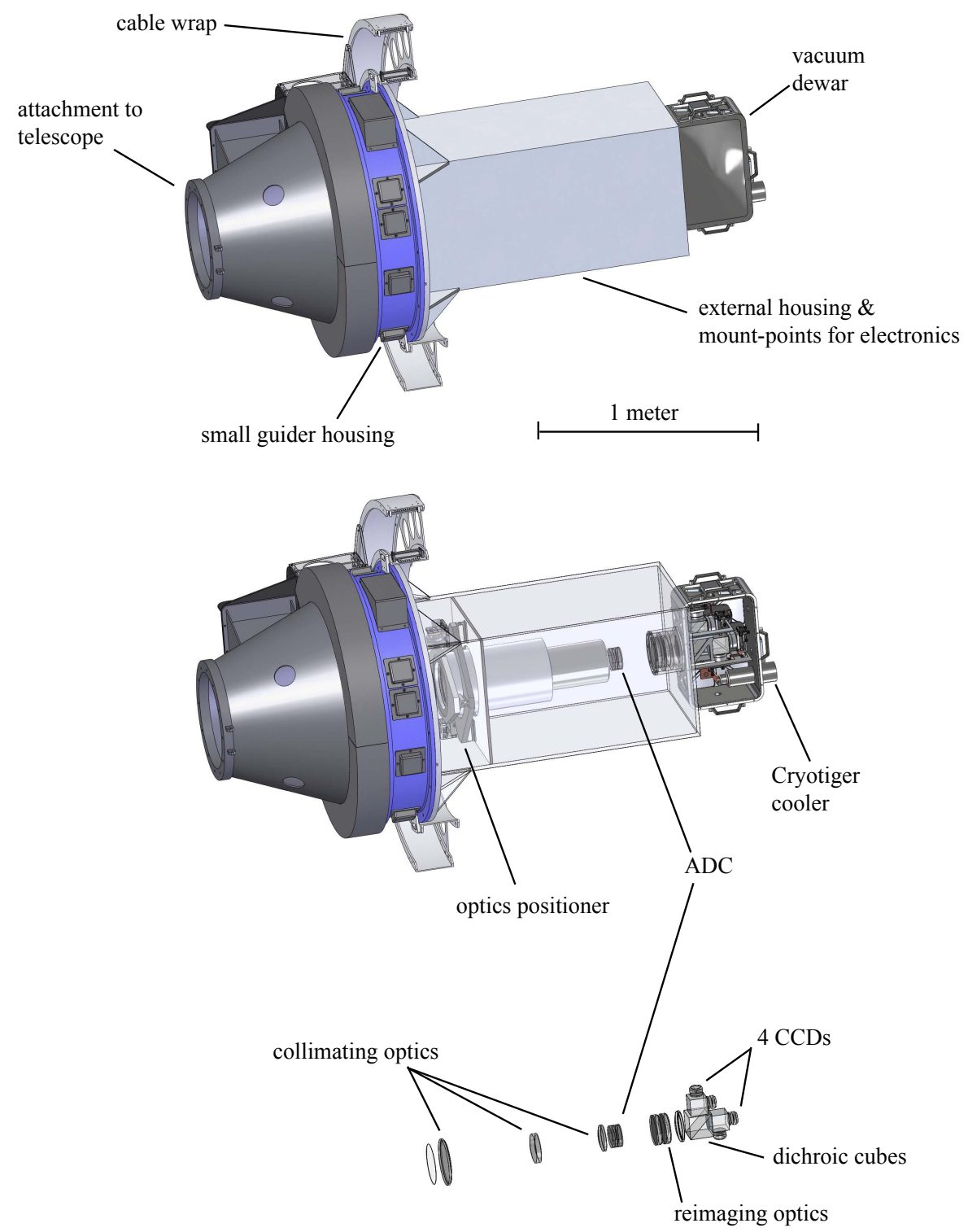

Figure 3. The design of the PISCO simultaneous multi-band imager, with structure removed to show the optical components. The telescope focal surface is collimated by three lenses whose alignment is computer controlled to compensate for structural deformities. Re-imaging optics produce a telecentric beam that is dissected by dichroic surfaces embedded in prisms to produce simultaneous images at $g^{\prime}, r^{\prime}, i^{\prime}$, and $z^{\prime}$ bands on four $3 \mathrm{~K} \times 6 \mathrm{~K}$ CCDs. There is an integral Atmospheric Dispersion Compensator and a Photometric Shutter. 


\subsection{Collimator Alignment}

In the event that PISCO is installed on a folded $\mathrm{f} / 11$ port on Magellan, a rigorous finite element analysis of the Magellan mounting flange revealed an unavoidable sag with respect to the optical axis due to the weight of PISCO plus the small guider housing system. This would result in a noticable degradation of image quality during periods of good seeing, so mitigation was accomplished by putting actuators on the collimator assembly internal to PISCO. This system will move the collimator in 4 axes by up to $3 \mathrm{~mm}$ in order to recenter the Magellan beam with the instrument. Since the beam emerging from the collimator is parallel, the subsequent optics in the dewar can sag somewhat without affecting image quality.

\subsection{Cryostat Internals}

The 18 inch x 12 inch x 10 inch cryostat enclosure houses the remaining optics, dichroics, and CCDs with the associated supporting thermal system. The window is a 6-inch lens of SFPL-53 that is the third lens of the imager. It is housed in a stainless steel lens cell constrained by teflon-tipped set screws and a Viton O-ring. All remaining optics are directly registered and fixed to this lens cell via direct mount of the dichroic prism cell and a titanium plate that serves as an optical bench for the filters, field lenses, and CCD. Only the CCDs themselves are cooled, although all the cryostat optics are in vacuum. The cryostat has weight-lightening pockets in its walls, and feedthrough ports for a vacuum pump, ion pump, vacuum gauge, CCD readout, thermal sensing/control, and cryogenic system. The CCD cooling to $\sim-100^{\circ} \mathrm{C}$ is accomplished with a Polycold compact compressor (PCC) system using a PT-30 gas mixture and high-performance cold head. Four 50-foot flexible PCC lines and two 25-foot lines connect the compressor (below the telescope room) and cold head within the cryostat. The cold head is coupled via copper straps and bars to a large copper plate below the titanium plate. The CCDs are coupled to the copper plate with individual copper straps and bars. Thermal control for each CCD is accomplished by a closed feedback loop using Lakeshore 325 units, platinum RTD sensors, and $50 \Omega$ power resistor heaters. An Agilent miniature ion pump extends the vacuum holding time to months.

\section{ELECTRONICS}

Electronic control of the collimator actuators, ADC system, photometric shutter, cryostat sensing/control, and readout system is housed in four thermally-insulated boxes, mounted to the sides of the main instrument structure. Servo-controlled fans circulate cooling within these insulated boxes and the heat is removed by radiators inside the boxes and tranferred to an external glycol loop provided by the observatory. It is important not to release any significant amount of heat into the telescope dome. The power supplies, actuator controllers and various safety and diagnostic sensors are contained within these boxes. The power switching and operation of all these system are controlled by a single low power (5 Watt) Diamond Systems Athena II computer which connects to the network and is the only system that boots on first application of power.

\subsection{Detectors}

PISCO utilizes 4 fully-depleted, $45 \mu \mathrm{m}$ thick MITLL CCID34 CCD detector arrays (one for each band), in a $3072 \times 614410 \mu \mathrm{m}$ pixel format with two amplifiers. The CCID34 design is a $10 \mu \mathrm{m}$ pixel pitch version of the CCID20 ${ }^{9}$ design used in several astronomical instruments. ${ }^{10,11}$ These devices have impressive QE in both blue and red, sometimes exceeding $86 \%$ at $450 \mu \mathrm{m}$ and $45 \%$ at $950 \mu \mathrm{m}$ and have extremely low read-noise $(\sim 2 \mathrm{e})$ and low dark current $(<3 \mathrm{e} /$ hour at $-120 \mathrm{C})$. We optimize the temperature of each CCD to get dark plus read noise equivalent to the expected best sky noise level in each band.

\subsection{Readout System}

The CCD readout system is based on the system designed by John Geary, and used in various instruments, including Megacam, MMIRS, and Kepler. We read out all 8 amplifiers (2 per CCD) at $500 \mathrm{kHz}$ translating to about 40 seconds, unbinned. 


\section{SOFTWARE}

Our software architecture is implemented in $\mathrm{C}++$. The instrument control is done via a Linux on the Athena II computer, and is network controlled with either xterm via ssh or direct TCP/IP socket command. The CCD read out is controlled with a rack-mounted Digilant $3 \mathrm{U}$ server running Linux located in a closet below the telescope, connected to the Geary CCD electronics via direct optical fiber.

\subsection{Data Reduction Pipeline}

Our cluster redshift determination method uses primarily the red sequence galaxies. Bright red galaxies make up most of the cluster population at any redshift. In a color-magnitude diagram they stand out as a striped band of galaxies (the "red sequence"), and their redshift is a monotonic function of their color as measured in $g-r, r-i$ or $i-z$, as appropriate for the redshift of the cluster galaxies. Since they are the brightest objects in every cluster, we extract only the most luminous resolved objects in each color bin.

We employ a novel technique called Stellar Locus Regression, ${ }^{12}$ that uses the locus of foreground galactic stars in color-color space to derive precise colors for the target galaxies in near-real time, We use SLR to correct for the majority of time-consuming impediments to ground-based optical astronomy. This includes corrections for aperture, atmospheric and galactic extinction, for non-photometric conditions. An additional advantage is that it eliminats the need to observe photometric standard fields each night. The image reduction is currently accomplished by a pipeline called PHOTPIPE ${ }^{13}$ which is employed in several previous projects, including SuperMACHO, ESSENCE, and SPT. PHOTOPIPE is highly customizable and parallelized and can create pixel masks, correct for crosstalk, debias, flat-field, and correct for non-uniform illumination in a few seconds. It then passes each image stack to the SLR routine for calibration and finally to the red-sequence cluster finder. This allows the determination, at the telescope and essentially in real time, whether enough photons have been collected to for a reliable photo- $z$ estimate. Once enough photons have been detected and a photo- $z$ has been determined, an automated slew to the next target is initiated.

\section{PERFORMANCE}

In the lab, we tested various aspects of the instrument. The total system gain, signal linearity, full-well level, and noise performance for each CCD was measured by a standard photon transfer curve procedure. The relative focus between each CCD is verified with an array of pin holes at the telescope focus. The total throughput of PISCO (Figure 4) was measured with an integrating sphere output near the telescope focus fed via optical fiber bundle from a monochromator and input intensity was measured by photodiode.

\section{ACKNOWLEDGMENTS}

Construction of PISCO was funded by NSFgrants: MRI-0723073, AST-1009649, and AST-1009012 with additional support from the CfA director's office. We wish to thank the staff at Harvard, Smithsonian, Carnegie, Magellan, and Las Campanas Observatories for their helpful support thoughout the development of PISCO. We also thank Mike Carr at Sci-In Tech for the photometric shutter and Astrodon Optics for the filter set. We thank Barr Precision Optics for producing the unique dichroic optics, and Harold Johnson Optical Laboratories for fabricating the lens elements. Our machinists at SAO and Harvard, Larry Knowles, Steve Sansone, Robert Snee, and Stanley Kench were indispensible resources in the construction and design. We thank Peter Doherty and Barry Burke for their expertise with CCDs and Armin Rest for developing the data reduction pipeline framework.

\section{REFERENCES}

[1] Carlstrom, J. E., Ade, P. A. R., Aird, K. A., Benson, B. A., Bleem, L. E., Busetti, S., Chang, C. L., Chauvin, E., Cho, H.-M., Crawford, T. M., Crites, A. T., Dobbs, M. A., Halverson, N. W., Heimsath, S., Holzapfel, W. L., Hrubes, J. D., Joy, M., Keisler, R., Lanting, T. M., Lee, A. T., Leitch, E. M., Leong, J., Lu, W., Lueker, M., Luong-van, D., McMahon, J. J., Mehl, J., Meyer, S. S., Mohr, J. J., Montroy, T. E., Padin, S., Plagge, T., Pryke, C., Ruhl, J. E., Schaffer, K. K., Schwan, D., Shirokoff, E., Spieler, H. G., Staniszewski, Z., Stark, A. A., Tucker, C., Vanderlinde, K., Vieira, J. D., and Williamson, R., "The 10 Meter South Pole Telescope," PASP 123, 568-581 (May 2011). 


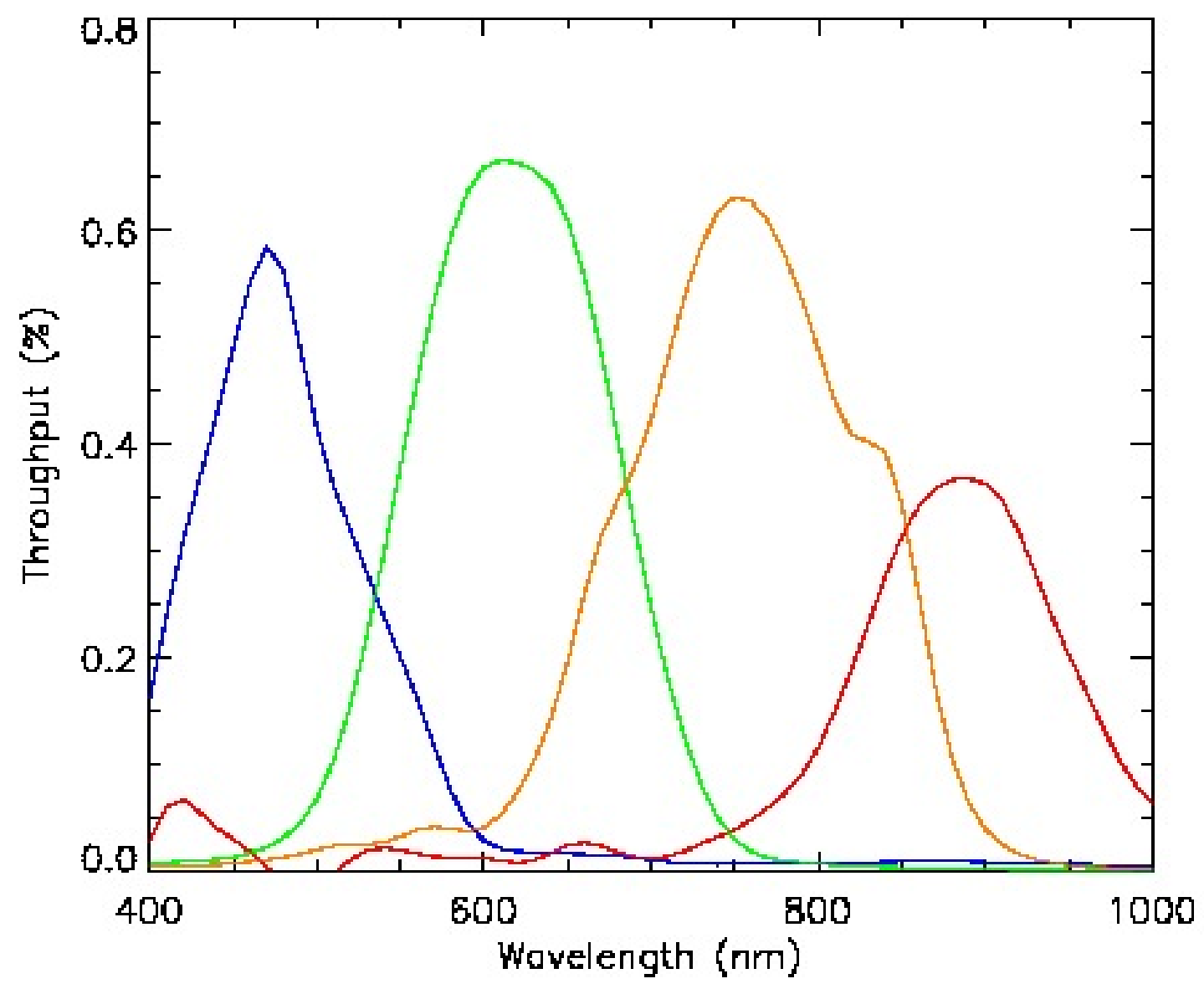

Figure 4. Throughput curves of the 4 bandpasses of PISCO without band-defining filters. 
[2] Padmanabhan, N., Hirata, C. M., Seljak, U., Schlegel, D. J., Brinkmann, J., and Schneider, D. P., "Correlating the CMB with luminous red galaxies: The integrated Sachs-Wolfe effect," PhysRevD 72, 043525 (Aug. 2005).

[3] Bahcall, N. A., Dong, F., Hao, L., Bode, P., Annis, J., Gunn, J. E., and Schneider, D. P., "The Richnessdependent Cluster Correlation Function: Early Sloan Digital Sky Survey Data," ApJ 599, 814-819 (Dec. 2003).

[4] Gladders, M. D. and Yee, H. K. C., "The Red-Sequence Cluster Survey. I. The Survey and Cluster Catalogs for Patches RCS 0926+37 and RCS 1327+29," ApJS 157, 1-29 (Mar. 2005).

[5] Song, J., Zenteno, A., Stalder, B., Desai, S., Bleem, L. E., Aird, K. A., Armstrong, R., Ashby, M. L. N., Bayliss, M., Bazin, G., Benson, B. A., Bertin, E., Brodwin, M., Carlstrom, J. E., Chang, C. L., Cho, H. M., Clocchiatti, A., Crawford, T. M., Crites, A. T., de Haan, T., Dobbs, M. A., Dudley, J. P., Foley, R. J., George, E. M., Gettings, D., Gladders, M. D., Gonzalez, A. H., Halverson, N. W., Harrington, N. L., High, F. W., Holder, G. P., Holzapfel, W. L., Hoover, S., Hrubes, J. D., Joy, M., Keisler, R., Knox, L., Lee, A. T., Leitch, E. M., Liu, J., Lueker, M., Luong-Van, D., Marrone, D. P., McDonald, M., McMahon, J. J., Mehl, J., Meyer, S. S., Mocanu, L., Mohr, J. J., Montroy, T. E., Natoli, T., Nurgaliev, D., Padin, S., Plagge, T., Pryke, C., Reichardt, C. L., Rest, A., Ruel, J., Ruhl, J. E., Saliwanchik, B. R., Saro, A., Sayre, J. T., Schaffer, K. K., Shaw, L., Shirokoff, E., Šuhada, R., Spieler, H. G., Stanford, S. A., Staniszewski, Z., Stark, A. A., Story, K., Stubbs, C. W., van Engelen, A., Vanderlinde, K., Vieira, J. D., Williamson, R., and Zahn, O., "Redshifts, Sample Purity, and BCG Positions for the Galaxy Cluster Catalog from the First 720 Square Degrees of the South Pole Telescope Survey," ApJ 761, 22 (Dec. 2012).

[6] Kotani, T., Kawai, N., Yanagisawa, K., Watanabe, J., Arimoto, M., Fukushima, H., Hattori, T., Inata, M., Izumiura, H., Kataoka, J., Koyano, H., Kubota, K., Kuroda, D., Mori, M., Nagayama, S., Ohta, K., Okada, T., Okita, K., Sato, R., Serino, Y., Shimizu, Y., Shimokawabe, T., Suzuki, M., Toda, H., Ushiyama, T., Yatsu, Y., Yoshida, A., and Yoshida, M., "MITSuME-Multicolor Imaging Telescopes for Survey and Monstrous Explosions," Nuovo Cimento C Geophysics Space Physics C 28, 755 (July 2005).

[7] Dunham, E. W., Elliot, J. L., Bida, T. A., Collins, P. L., Taylor, B. W., and Zoonematkermani, S., "HIPO data products," in [Society of Photo-Optical Instrumentation Engineers (SPIE) Conference Series], Society of Photo-Optical Instrumentation Engineers (SPIE) Conference Series 7014 (Aug. 2008).

[8] Greiner, J., Bornemann, W., Clemens, C., Deuter, M., Hasinger, G., Honsberg, M., Huber, H., Huber, S., Krauss, M., Krühler, T., Küpcü Yoldaş, A., Mayer-Hasselwander, H., Mican, B., Primak, N., Schrey, F., Steiner, I., Szokoly, G., Thöne, C. C., Yoldaş, A., Klose, S., Laux, U., and Winkler, J., "GROND-a 7-Channel Imager," PASP 120, 405-424 (Apr. 2008).

[9] Burke, B. E., Gregory, J. A., Mountain, R. W., Kosicki, B. B., Savoye, E. D., Daniels, P. J., Dolat, V. S., Lind, T. L., Loomis, A. H., Young, D. J., Luppino, G. A., and Tonry, J. L., "Large-Area BackIlluminated CCD Imager Development," in [Optical Detectors for Astronomy], Beletic, J. and Amico, P., eds., Astrophysics and Space Science Library 228, 19 (1998).

[10] Cuillandre, J.-C., Luppino, G. A., Starr, B. M., and Isani, S., "Performance of the CFH12K: a 12K by 8K CCD mosaic camera for the CFHT prime focus," in [Optical and IR Telescope Instrumentation and Detectors], Iye, M. and Moorwood, A. F., eds., Society of Photo-Optical Instrumentation Engineers (SPIE) Conference Series 4008, 1010-1021 (Aug. 2000).

[11] Clemens, J. C., Crain, J. A., and Anderson, R., "The Goodman spectrograph," in [Ground-based Instrumentation for Astronomy], Moorwood, A. F. M. and Iye, M., eds., Society of Photo-Optical Instrumentation Engineers (SPIE) Conference Series 5492, 331-340 (Sept. 2004).

[12] High, F. W., Stubbs, C. W., Rest, A., Stalder, B., and Challis, P., "Stellar Locus Regression: Accurate Color Calibration and the Real-Time Determination of Galaxy Cluster Photometric Redshifts," AJ 138, 110-129 (July 2009).

[13] Rest, A., Stubbs, C., Becker, A. C., Miknaitis, G. A., Miceli, A., Covarrubias, R., Hawley, S. L., Smith, R. C., Suntzeff, N. B., Olsen, K., Prieto, J. L., Hiriart, R., Welch, D. L., Cook, K. H., Nikolaev, S., Huber, M., Prochtor, G., Clocchiatti, A., Minniti, D., Garg, A., Challis, P., Keller, S. C., and Schmidt, B. P., "Testing LMC Microlensing Scenarios: The Discrimination Power of the SuperMACHO Microlensing Survey," ApJ 634, 1103-1115 (Dec. 2005). 